\title{
Finite-Difference Time-Domain Simulations of Radon Transport in Porous Media
}

\author{
A. Tayebi ${ }^{1^{\star}}$, H. Bezzout ${ }^{2}$, M. El Maghraoui ${ }^{1}$ and H. El Faylali ${ }^{2}$ \\ ${ }^{I}$ Laboratory of Optoelectronics, Physical Chemistry Materials and Environmental, \\ Faculty of Sciences, Ibn Tofail University, PB 133 Kenitra, Morocco \\ ${ }^{2}$ Laboratory of Computer Science, Systems and Optimization (Informatics, Systems and Optimization), \\ Faculty of Sciences, Ibn Tofail University, PB 133 Kenitra, Morocco
}

\section{ARTICLE INFO}

Article history:

Received 4 November 2018

Received in revised form 28 July 2020

Accepted 15 September 2020

Keywords:

Radon

Radon transport equation

Finite-difference time domain (FDTD)

Diffusion coefficient

\begin{abstract}
A B S T R A C T
In this work, an efficient algorithm, using a finite-difference time-domain (FDTD) technique, is proposed for modeling the variation of radon concentration as a function of soil structure parameters and vice versa. The development of the FDTD model is based on the simultaneous resolution of the radon transport equation in a porous, homogeneous medium, namely the soil. This equation describes the concentration of radon per pore volume unit. The numerical results are compared with those of the literature or with the theoretical ones.
\end{abstract}

\section{INTRODUCTION}

Radon from soil and rocks is released into the atmosphere and becomes the most important factor influencing exposure to naturally-occurring radiation $[1,2]$. More than $80 \%$ of the radon emitted into the atmosphere comes from soil and rocks on the surface of the earth [3]. However, in most cases, the dominant transport mechanism is the diffusion caused by the radon concentration gradient between these media and atmospheric air [4], as shown in Fig. 1.

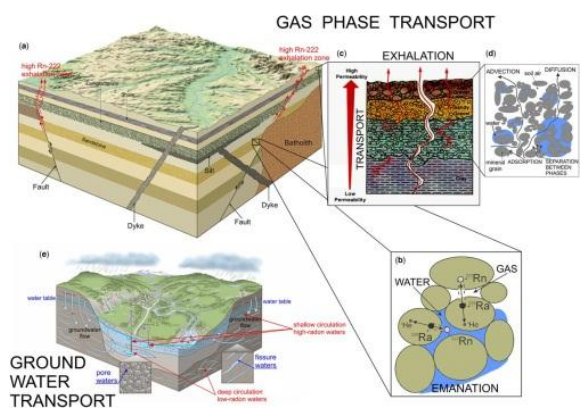

Fig. 1. llustration of the process of ${ }^{222} \mathrm{Rn}$ release from the lithosphere into the atmosphere.

${ }^{*}$ Corresponding author.

E-mail address: abderrahim.tayebi@ uic.at.ma

DOI: https://doi.org/10.17146/aij.2020.902
In the field of radon intrusion, a range of analytical and numerical models have been developed [5-8]. Several two-dimensional analytical models have been developed recently $[5,9]$. The widely-used analytical models, the JohnsonEttinger model [10], the Volasoil model [11], and the improved Volasoil model [12], evaluate the entry of pollutants in relation to the transport mechanism.

The finite-difference time-domain (FDTD) technique has been successfully applied by many authors to analyze problems with complex materials [13]. FDTD is capable of achieving arbitrary accuracy that is only limited by the choice of the numerical grid size in time and space. The approach based on the auxiliary differential equation (ADEFDTD) was used in modeling of electromagnetic wave propagation in magnetized plasma [14], analysis of the light extraction efficiency of OLEDs with a random scattering layer [15], lightning electromagnetic pulse and surge simulations [16], and computation of lightning horizontal field over two and three dimensions [17]. The Z-transform technique combined with the FDTD method gave consistent results for the simulation of solitons in nonlinear dispersive media $[18,19]$. In order to simulate the wave's propagation in dispersive media with complex permittivity, methods based on 
the discrete convolution of the dispersion relation are presented in [20]. The alternating-direction implicit finite-difference time-domain (ADI-FDTD) technique has been introduced as an unconditionally stable implicit method to solve the problems of EM radiation in nonlinear dispersive media [21].

In this paper, developed was a new algorithm based on the FDTD method to model the variation of radon concentration as a function of soil structure parameters and vice versa. This model describes the space evolution of concentration of radon per pore volume unit. Furthermore, the simulation's results are compared to those of the literature or obtained by the theoretical solutions.

\section{THEORY}

The level of emanation, at a point of a soil matrix due to a radon source, depends on such factors as its width, its depth, and the nature of the soil that covers it. Here, the transport of radon at a source covered by a layer of soil will be modeled.

The phenomenon of radon diffusion in a porous medium (soil) is described by the Fick's law $[22,23]$, as given by (1):

$$
\vec{J}=-D_{e} \vec{\nabla} N
$$

In (1), $D_{e}=D / \varepsilon, N$ is the per-unit-pore-volume concentration, and $D$ is the radon molecular diffusion coefficient in the fluid (liquid or gas) filling the pores of the medium in question $\left(\mathrm{m}^{2} \mathrm{~s}^{-1}\right), \varepsilon$ is the soil porosity, and $J$ is the density of the diffused flux per unit area of the pores. Taking into account diffusion transport, the limit of migration of radon into the soil is about $2 \mathrm{~m}$ [24].

However, radon can come from very deep sources, about $100 \mathrm{~m}$ [24].This is due to convection transport: radon is driven by interstitial fluids, moving in fractures and micro fractures of the soil. This convection transport is governed by Darcy's law [23], as given in (2).

$$
\vec{v}=-\frac{k}{\mu} \vec{\nabla} P
$$

In (2), $v$ is the Darcy velocity in $\mathrm{m} \mathrm{s}^{-1}, k$ is the intrinsic permeability of the soil expressed in $\mathrm{m}^{2}, P$ is the intrinsic fluid pressure in $\mathrm{Pa}$, and $\mu$ is the dynamic viscosity of the fluid.

The diffused radon flux per unit area due to diffusion (1) and convection (2) can therefore be expressed by (3).

$$
\vec{J}=-D_{e} \vec{\nabla} N-\frac{k}{\mu} N \vec{\nabla} P=-D_{e} \vec{\nabla} N+V N
$$

If we consider an element of volume $d V$ limited by a surface $S$, the equilibrium in volume $V$ is described by the expression (4).

$$
\iint_{S} \vec{j} \vec{n} d s+\iiint_{V} \lambda N d V-\iiint_{V} Q d V=E
$$

with $E=-\iint_{V} \int \frac{\partial N}{\partial t} d V$

In (4), $\lambda$ is the decay constant of radon-222 = $2.07 \times 10^{-6} \mathrm{~s}^{-1}$ and $Q$ is the number of radon atoms created per cubic-meter per second.

Applying the divergence theorem to equation (4) results in (5).

$$
\operatorname{div}\left(-\frac{D}{\varepsilon} \vec{\nabla} N+V N\right)+\lambda N-Q=-\frac{\partial N}{\partial t}
$$

The transport of radon is expressed mathematically by the time equation after the development of equation (5), which is composed of two phenomena: diffusion and advection [24]. Equation (6) equation describes the transport of radon in space and time:

$$
\frac{\partial N}{\partial t}=D_{e} * \nabla^{2} N-V \cdot \nabla N-\lambda N+Q
$$

where

$$
\nabla^{2} N=\left(\frac{\partial N}{\partial x}\right)^{2}+\left(\frac{\partial N}{\partial y}\right)^{2}+\left(\frac{\partial N}{\partial z}\right)^{2}
$$

Equation (7) is simplified as a two-dimensional (2D) equation presented in (8).

$$
\frac{\partial N}{\partial t}=D_{e}\left(\left(\frac{\partial N}{\partial x}\right)^{2}+\left(\frac{\partial N}{\partial y}\right)^{2}\right)-F
$$

with $F=V\left(\frac{\partial N}{\partial y}\right)+\lambda N$

Figure 2 describes the configuration of radon transport from the depth to the surface. Here, $a$ is the width and $b$ is the depth of this configuration.

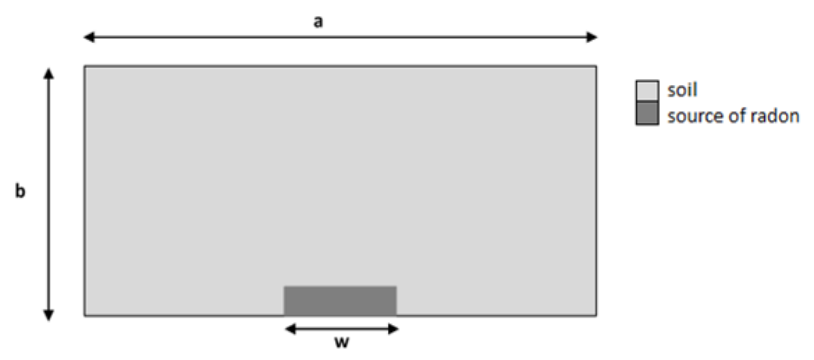

Fig. 2. Space configuration of radon transport. 
The FDTD method based on the discretization of the partial equation in time and space is adopted to solve this 2-D equation.

From (8), (9) is obtained.

$$
\left.\mathbf{N}\right|_{i, j} ^{n+1}=\left.\mathbf{N}\right|_{i, j} ^{n}+A+B-C-D
$$

where:

$$
\begin{aligned}
A & =D_{e} \frac{\Delta t}{(\Delta x)^{2}}\left(\left.\mathbf{N}\right|_{i+1, j} ^{n}-\left.2 \mathbf{N}\right|_{i, j} ^{n}-\left.\mathbf{N}\right|_{i-1, j} ^{n}\right) \\
B & =D_{e} \frac{\Delta t}{(\Delta y)^{2}}\left(\left.\mathbf{N}\right|_{i, j+1} ^{n}-\left.2 \mathbf{N}\right|_{i, j} ^{n}-\left.\mathbf{N}\right|_{i, j-1} ^{n}\right) \\
C & =V \frac{\Delta t}{\Delta x}\left(\left.\mathbf{N}\right|_{i, j} ^{n}-\left.\mathbf{N}\right|_{i-1, j} ^{n}\right) \\
D & =\left.\lambda \Delta t \mathbf{N}\right|_{i, j} ^{n}
\end{aligned}
$$

In the equation (9), the FDTD is implemented [25] by combining a forward central difference for a temporal discretization and a centralization for space discretization.

The boundary conditions of the problem may be written as in (10-12).

$$
\begin{aligned}
& N(x=0)=0 \\
& N(x=a)=0 \\
& N(x)=N_{0}\left\{\begin{array}{l}
\text { if } x<\frac{(a+w)}{2} \\
\text { if } x>\frac{(a-w)}{2}
\end{array}\right.
\end{aligned}
$$

Figure 3 shows us the algorithm to implement in a high-level language programming. In this work, the Python language was chosen as a tool to develop the solution to this problem because of its robustness and simplicity in providing a powerful program.

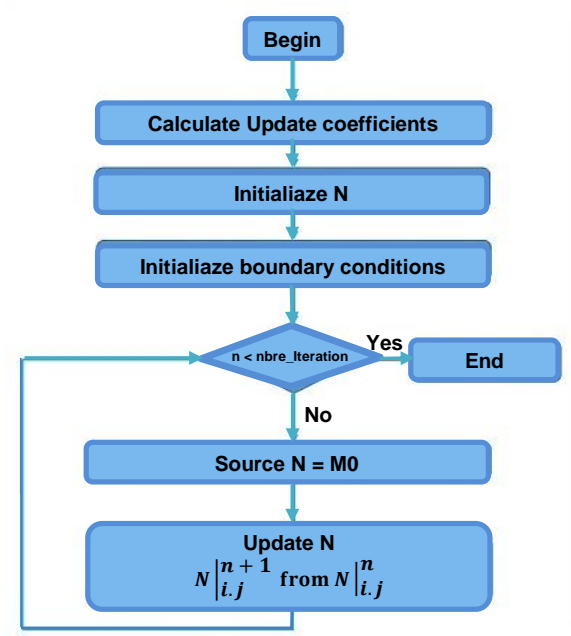

Fig. 3. Algorithm diagram to implement radon transport with FDTD method.

\section{RESULTS AND DISCUSSION}

\section{Influence of diffusion coefficient}

Figure 4 shows the distribution of the radon concentration, normalized to the source concentration $N_{0}$, at a depth of $0.7 \mathrm{~m}$ for different diffusion coefficients.

The parameters involved in the radon propagation equation are as follows: $b=2.8 \mathrm{~m}$; $w=6.5 \mathrm{~m} ; V=0 ; a=26 \mathrm{~m}$.

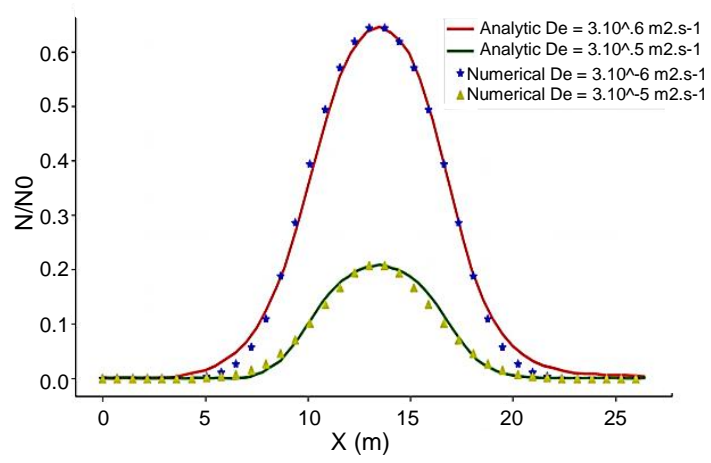

Fig . 4. Calculation of radon concentration variation at a source for different diffusion coefficients.

It can be deduced from Fig. 4 that:

- The peak of radon corresponds to the middle of the source in the FDTD and analytical curves $(x=a / 2)$

- The height of the peak increases with the diffusion coefficient (soil type) in the same way for the FDTD curves and the analytical curves.

- The half-height width of the radon peak is the width of the source. This allows us to predict the width of the source (fault) from the experimental results.

It can be concluded, from this first result where the diffusion coefficient (soil type) is varied, that the numerical method FDTD attains results that perfectly match the analytical results with an extremely fast computing speed.

\section{Influence of depth}

Figure 5 shows the variation of the calculated concentration at different depths. The emanation rate increases with depth, which means that when measuring the radon level in the field, the measuring stations must be placed at the same depth. In addition, the probability of detection of a source increases if the concentration of radon is measured at a greater depth.

The parameters involved in the radon propagation equation are as follows:

$$
D_{e}=3 \times 10^{-6} \mathrm{~m}^{2} \mathrm{~s}^{-1} ; w=6.5 \mathrm{~m} ; V=0 ; a=26 \mathrm{~m} .
$$




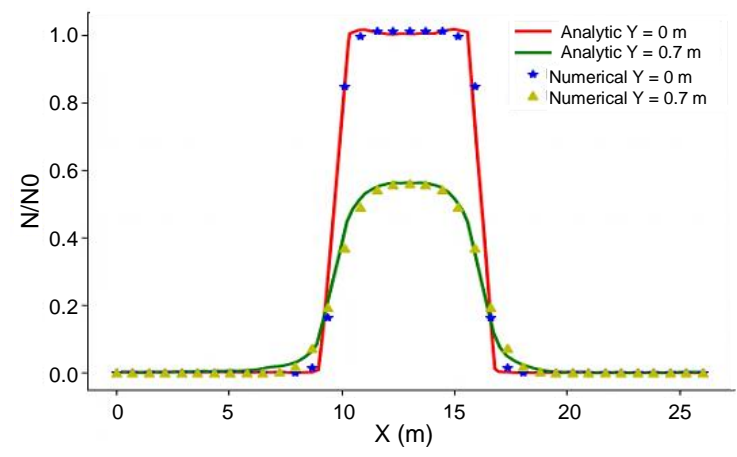

Fig. 5. Variation of radon concentration at a source for different depths.

Once again, the curves of the FDTD method perfectly match those of the analytical method.

\section{Influence of the width of the fault}

Figure 6 shows the variation of the radon concentration at a depth of $0.7 \mathrm{~m}$ for different widths $w$ of the source. It is observed that the concentration of radon increases with the width of the fault.

The parameters involved in the radon propagation equation are as follows:

$$
D_{e}=3 \times 10^{-6} \mathrm{~m}^{2} \mathrm{~s}^{-1} ; b=2.8 \mathrm{~m} ; V=0 ; a=26 \mathrm{~m} .
$$

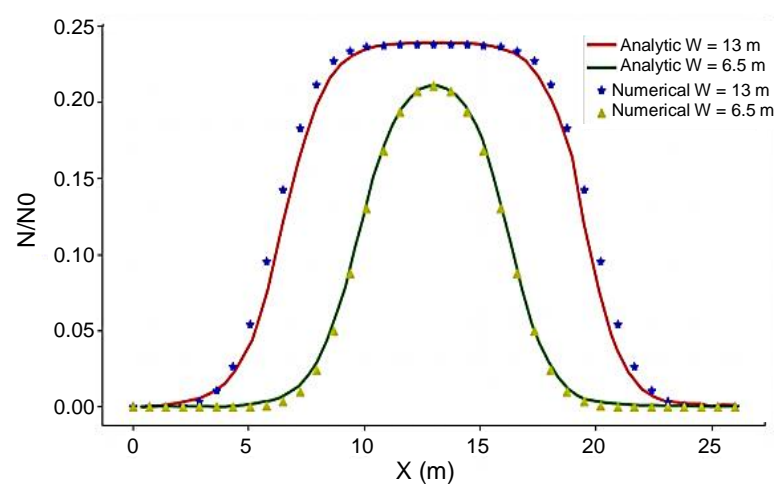

Fig. 6. Radon concentration variation for different widths of the source.

Again, when varying the width of the sources, the curves of the FDTD method perfectly match the results of the analytical method.

By varying the different parameters of the radon propagation equation by the numerical method FDTD, the results obtained for conditions similar to that of the analytical method were duplicated. A digital-analytical comparison made it possible to validate the reliability of the simulation technique used for electromagnetic waves (FDTD) in order to use it for the study of the propagation of radon in the ground, which opens up the possibility of studying the transport of the latter on a three-dimensional scale and personalized precision.

\section{CONCLUSION}

After comparing the results of the FDTD numerical method developed in this work to that of the result of analytical method by varying the three key parameters of the radon propagation equation, namely the diffusion coefficient and the depth and the width of the source, the reliability of the numerical method can be validated.

This digital FDTD method will allow the interpretation of the soil information that can be provided by measuring the radon concentration at a given depth with good accuracy and speed of calculation.

\section{ACKNOWLEDGMENT}

The authors warmly thank the Laboratory of Optoelectronics, Physical Chemistry of Materials and the Environment of the Faculty of Science, Ibn Tofail University, and the Laboratory of Computer Science, Systems and Optimization of the Faculty of Science, Ibn Tofail University.

\section{REFERENCES}

1. J. Yang, H. Busen, H. Scherb et al., Sci. Total Environ. 656 (2019) 1304.

2. A.F. Saad, R.M. Abdallah and N.A. Hussein, Appl. Radiat. Isot. 137 (2018) 273.

3. D.E. Tchorz-Trzeciakiewicz and M. Kłos, Sci. Total Environ. 584 (2017) 911.

4. M.P. Campos, L.J.P. Costa, M.B. Nisti et al., J. Environ. Radioact. 172 (2017) 232.

5. S. Chakraverty, B.K. Sahoo, T.D. Rao et al., J. Environ. Radioact. 182 (2018) 165.

6. R. Shen, K.G. Pennell and E.M. Suuberg. Chemosphere 95 (2014) 140.

7. Y.-J. Ye, X.-T. Dai, D.-X. Ding et al., J. Environ. Radioact. 165 (2016): 219.

8. N. Chauhan and R.P. Chauhan, J. Environ. Radioact. 144 (2015) 57.

9. R. Shen and E.M. Suuberg, Environ. Modelling \& Software 54 (2014) 1.

10. P.C. Johnson and R.A. Ettinger, Environ. Sci. Technol. 25 (1991) 1445.

11. M.F.W. Waitz, J.I. Freijer, P. Kreule et al., The VOLASOIL risk assessment model based on CSOIL for soils contaminated with volatile compounds (1996). 
12. J. Bakker, J.P.A. Lijzen and H.J. van Wijnen, Site-specific human risk assessment of soil contamination with volatile compounds, Bilthoven, The Netherlands: RIVM (2008).

13. Md. Gaffar and D. Jiao, IEEE Transactions on Microwave Theory and Techniques 62 (2014) 2538.

14. A. Samimi and J.J. Simpson, IEEE Transactions on Antennas and Propagation 63 (2014) 269.

15. J.W. Kim, J.H. Jang, M.C. Oh et al., Optics Express 22 (2014) 498.

16. S. Imato, Y. Baba, N. Nagaoka et al., IEEE Transactions on Electromagnetic Compatibility 58 (2016).

17. Q. Zhang, D. Li, X. Tang et al., IEEE Transactions on Electromagnetic Compatibility 55 (2013).
18. T. Wang, Z. Zhang, F. Liao et al., Scientific Reports 4 (2014) 4052.

19. C. Varin, R. Emms, G. Bart et al., Comput. Phys. Commun. 222 (2018) 70.

20. W.J. Chen, W. Shao, H. Chen et al., IEEE Microwave and Wireless Components Letters 24 (2013) 75 .

21. J.P. Wilson and W. Dai, Comput. Phys. Commun. 235 (2019) 279.

22. M. Culot, H. Olson and K. Schiager, Health Phys. 30 (1976).

23. W. Nazaroff, Reviews of Geophysics 30 (1992) 137.

24. R. Catalano, G. Imme, G. Mangano et al., Radiation Protection Dosimetry (2015) 1.

25. M.O.F. Howlader and T.P. Sattar, Progress in Electromagnetics Research 44 (2015) 127. 